\title{
Appetite-regulating hormones in early life and relationships with type of feeding and body composition in healthy term infants
}

\author{
Laura M. Breij ${ }^{1}$ Monique T. Mulder ${ }^{2}$ Leonie C. van Vark-van der Zee ${ }^{2}$. \\ Anita C. S. Hokken-Koelega ${ }^{1}$
}

Received: 8 February 2016 / Accepted: 25 April 2016 / Published online: 11 May 2016

(C) The Author(s) 2016. This article is published with open access at Springerlink.com

\begin{abstract}
Introduction Body composition in early life influences development of obesity during childhood and beyond. Appetite-regulating hormones (ARH) play a role in regulation of food intake and might thus influence body composition in later life. Studies on associations between ARH and body composition in early life are limited.

Methods In 197 healthy term infants, we measured serum fasting levels of ghrelin, leptin, insulin, glucose-dependent insulinotropic peptide (GIP), pancreatic polypeptide (PP) and peptide YY (PYY) at 3 months and in 41 infants also at 6 months and their associations with type of feeding and longitudinal fat mass percentage (FM\%) measured by air displacement plethysmography at 1,3 and 6 months and abdominal visceral and subcutaneous fat, measured by ultrasound, at 3 and 6 months.

Results Infants with formula feeding for 3 months had significantly higher serum levels of ghrelin, leptin, insulin, GIP and PP ( $p=0.026, p=0.018, p=0.002, p<0.001$, resp.) and lower serum levels of PYY $(p=0.002)$ at 3 months than breastfed infants. Leptin and ghrelin correlated positively with FM\% at 3 months and insulin with change in FM\% between 1 and 3 months $(r=0.40$, $p<0.001, r=0.23, p<0.05, r=0.22, p<0.01$, resp.).
\end{abstract}

Laura M. Breij

1.breij@erasmusmc.nl

1 Department of Pediatrics, Subdivision of Endocrinology, Erasmus Medical Center/Sophia Children's Hospital, Room Number: Sk-0152, Dr. Molenwaterplein 40, 3015 GJ Rotterdam, The Netherlands

2 Section of Pharmacology Vascular and Metabolic Diseases, Department of Internal Medicine, Cardiovascular Research School COEUR, Erasmus Medical Center, Rotterdam, The Netherlands
Leptin at 3 months correlated with subcutaneous fat at 3 months $(r=0.23, p<0.001)$, but not with visceral fat. Other ARH did not correlate with body composition.

Conclusion Formula-fed infants had a different profile of ARH than breastfed infants, suggesting that lower levels of ghrelin, leptin and insulin in breastfed infants contribute to the protective role of breastfeeding against obesity development. Leptin, ghrelin and insulin were associated with fat mass percentage or its changes.

Keywords Appetite-regulating hormones · Early life · Formula fed $\cdot$ Breastfed $\cdot$ Body composition

\section{Introduction}

Childhood obesity is a worldwide problem with an increasing prevalence of $7-11 \%$ in Dutch children aged 4-12 years [1]. It is not only associated with short-term morbidity, but also associated with long-term morbidity, such as adult obesity, type 2 diabetes and cardiovascular diseases [2-4]. Accelerated weight gain during the first 3 months of life is associated with accumulation of fat mass during childhood and a worse cardiovascular and metabolic profile in young adulthood [5-7]. Appetite-regulating hormones play a role in the regulation of food intake and body composition by signaling satiety and energy reserves through hypothalamic receptors [8]. However, little is known about the relation between body composition and appetite-regulating hormones in early life [9].

Changes in body composition might be influenced by programming of the orexigenic and anorectic appetiteregulating hormones [10]. Orexigenic appetite stimulating hormones, such as ghrelin, are important in the initiation, cessation and frequency of eating. Anorectic 
appetite-regulating hormones, such as leptin and peptide YY (PYY), decrease food intake and increase metabolic rate. The glucose-dependent insulinotropic peptide (GIP) stimulates pancreatic beta cells in response to the ingestion of meals or glucose $[11,12]$. All these signals act at several sites in the central nervous system (CNS), but the pathways converge to the hypothalamus, which contains a large number of peptides and other neurotransmitters that influence food intake.

The protective role of breastfeeding for obesity could be partly explained by the composition of the human milk, but probably also by different appetite-regulating hormones in infants due to breastfeeding. Knowledge about the changes in appetite-regulating hormones during infancy is very limited [13].

We hypothesized that formula-fed infants would have a different profile of serum appetite-regulating hormones than breastfed infants and that fasting serum ghrelin, leptin and insulin levels would be positively associated with gain in weight and in fat mass during the first 3 months after birth, while GIP, PP and PYY levels would be negatively associated. We, therefore, investigated fasting serum levels of ghrelin, leptin and insulin, GIP, PP and PYY at 3 months and associated these with type of feeding and body composition, including FM\% and visceral and subcutaneous fat at 3 and 6 months.

\section{Materials and methods}

\section{Subjects}

The current study is part of a birth cohort study (Sophia Pluto Study) which started in January 2013, aiming to provide detailed data on body composition and growth in early life.

Serum levels of appetite-regulating hormones (ghrelin, leptin, insulin, GIP, PYY and PP) were determined in a random subgroup of 197 infants at 3 months of age and in 41 of them also at 6 months of age.

Infants were recruited from several hospitals in and near Rotterdam, a large city in The Netherlands. All participants fulfilled the same inclusion criteria: (1) born term ( $\geq 37$ weeks of gestation), (2) age $<28$ days and (3) uncomplicated neonatal period without signs of severe asphyxia (defined as an Apgar score below three after $5 \mathrm{~min}$ ), sepsis or long-term complication of respiratory ventilation. Exclusion criteria were known congenital or postnatal diseases that could interfere with body composition development, confirmed intrauterine infection, maternal use of corticosteroids or significant maternal medical condition that could interfere with infant's body composition development (e.g., diabetes).
The Medical Ethics Committee of Erasmus Medical Center approved the study. Written informed consent was obtained from both parents unless mother was single.

\section{Data collection and measurements}

Information about the type of feeding was recorded during outpatient clinic visits.

\section{Infant characteristics}

Research clinic visits were scheduled at 1, 3 and 6 months. Birth data were taken from midwife and hospital records. Trained pediatric nurses took the measurements according to standard procedures.

Anthropometrics Weight was measured to the nearest gram by an electronic infant scale (Seca, Hanover, MD). Length was measured to the nearest $0.1 \mathrm{~cm}$ by a length meter (Seca). Head circumference was measured to the nearest $0.1 \mathrm{~cm}$ using measuring tape (Seca, circumeter). Weight SDS, height SDS and weight for length SDS were calculated with Growth Analyser Research Calculation Tools 4.0 (available at www.growthanalyser.org), according to Dutch age- and gender-matched reference values [14].

Body composition Whole-body composition was assessed using air displacement plethysmography (Peapod, Infant Body Composition System, COSMED). A detailed description of the air displacement plethysmography (ADP) system is provided elsewhere [15-18]. Briefly, this ADP system assesses fat mass (FM), fat mass percentage (FM\%) and fat-free mass (FFM) and fat-free mass percentage (FFM\%) by direct measurements of body volume and body mass, based on the whole-body densitometric principle. All measurements were obtained by experienced personnel, according to standardized protocol. The Peapod was calibrated every day, according to the protocol recommended by the supplier.

Abdominal fat Visceral and abdominal subcutaneous fat was measured at 3 and 6 months using a Prosound 2 ultrasound, with a UST-9137 convex ultrasound transducer (both from Hitachi Aloka Medical, Switzerland). For both measures, the transducer was positioned where the xiphoid line intercepted the waist circumference measurement plane. Visceral fat was estimated by measuring visceral depth, which is the distance between the peritoneal boundary and the corpus of the lumbar vertebra, assessed in the longitudinal plane with the ultrasound probe depth set at $9 \mathrm{~cm}$. Subcutaneous abdominal fat was estimated by the distance between the cutaneous boundary and the linea alba at the same location, but on a transverse plane with a probe depth of $4 \mathrm{~cm}$ [19]. 


\section{Collection of blood and assays}

At 3 months, blood samples were collected from a heel prick after the infants had fasted for at least $3 \mathrm{~h}$. To stabilize the appetite-regulating hormones, blood samples were collected in EDTA tubes and dipeptidyl peptidase-4 inhibitor (DPP4-inhibitor, Merck Chemicals) and 4-(2-aminoethyl)benzenesulfonyl fluoride hydrochloride (serine protease inhibitor, Calbiochem) were added at the time of collection. Blood was centrifuged at $4{ }^{\circ} \mathrm{C}$ to prepare plasma, which was quickly frozen. Samples were stored at $-80^{\circ} \mathrm{C}$.

Ghrelin (active), leptin, insulin, GIP, PP and PYY concentrations in serum were determined by the MILLIPLEX MAP Human Metabolic Hormone Magnetic Bead Panel, catalog number HMHMAG-34K (Millipore Corporation, Billerica, MA) using the commercial protocol. The intra-assay $\mathrm{CV}$ was $<10 \%$, and the inter-assay $\mathrm{CV}$ was $<15 \%$.

\section{Statistical analysis}

Descriptive results are expressed as median (interquartile range). Differences between groups were examined using Mann-Whitney $U$ tests. We assessed linear correlations between levels of ghrelin, leptin, insulin, GIP, PP and PYY and other parameters using Pearson's correlation coefficient. Correlation coefficient below 0.20 was considered to be a negligible correlation. SPSS statistical package version 20.0 (SPSS Inc. Chicago, Illinois) was used. All statistical tests were performed two-sided, and results were regarded statistically significant if the $P$ value was $<0.05$.

\section{Results}

Clinical characteristics of the infants are presented in Table 1. The median (IQR) gestational age was 39.9 (38.940.6) weeks, and $53 \%$ of the infants were boys. Forty-one boys had their appetite-regulating hormones longitudinally determined at 3 and 6 months. Fifty-six percentage of the infants received exclusive breastfeeding at 1 month, $32 \%$ at 3 months, and $16 \%$ at 6 months.

Table 2 shows serum levels of ghrelin, leptin, insulin, GIP, PP and PYY at 3 and 6 months. Median fasting time had been 3.00 (2:30-3:40) hours at 3 months and 2.52 (2:13-4:15) hours at 6 months. Ghrelin, GIP and PP levels increased significantly from 3 to 6 months $(p<0.001$, $p=0.016, p<0.001$, resp.), while the leptin levels decreased between 3 and 6 months $(p=0.009$ ).

There were no differences in appetite-regulating hormone levels between boys and girls (Table 2).

\section{Associations between appetite-regulating hormones and type of feeding}

Table 3 shows the serum levels of appetite-regulating hormones during exclusively formula feeding versus exclusively breastfeeding for 3 months. There were no significant differences in weight SDS, FM\%, visceral and subcutaneous fat at 1, 3 and 6 months between the formula-fed and breastfed infants, but all appetite-regulating hormones were different between the formula-fed and breastfed groups. Serum levels of ghrelin, leptin, insulin, GIP and PP were significantly higher in the formula-fed group compared to the breastfed group ( $p=0.026, p=0.018, p=0.002, p<0.001$, resp.), whereas PYY was significantly lower $(p=0.002)$.

At 3 months, a shorter duration of exclusive breastfeeding correlated only with a higher PP level $(r=-0.24$, $p=0.005$ ). At 6 months, we found no significant correlations of duration of breastfeeding with appetite-regulating hormones.

\section{Associations between appetite-regulating hormones and fat mass percentage}

Serum leptin at 3 months correlated with fat mass percentage $(\mathrm{FM} \%)$ at that age $(r=0.37, p<0.001)$ (Table 4$)$. Similar associations were found between serum leptin at 3 months and FM\% at 6 months $(r=0.41, p<0.001)$. Serum insulin at 3 months correlated with the increase in FM\% between 1 and 3 months $(r=0.22, p<0.01)$. The other appetite-regulating hormones did neither correlate with FM\% nor correlate with changes in FM\%. We found in the formula-fed infants a positive correlation between ghrelin at 3 months and FM\% $(r=0.23, p<0.05)$ at that age. In the breastfed infants, we did not find this correlation, but a stronger correlation between leptin and FM\% $(r=0.61, p<0.001)$ (data not shown).

\section{Associations between appetite-regulating hormones and visceral and subcutaneous fat}

Serum leptin, ghrelin and insulin at 3 months did not correlate with visceral and subcutaneous fat at 3 and 6 months. Breastfed infants showed a correlation between leptin and subcutaneous fat at 3 months $(r=0.38, p<0.01)$ (data not shown).

\section{Associations between appetite-regulating hormones and anthropometrics}

As a proxy for body composition, anthropometrics are often used. We, therefore, also analyzed correlations between appetite-regulating hormones and anthropometrics. Serum leptin at 3 months correlated positively with weight for length SDS at 3 months $(r=0.48, p<0.001)$ 
Table 1 Clinical characteristics

\begin{tabular}{llllll} 
Total group & $\begin{array}{l}\text { Girls } \\
(n=93)\end{array}$ & $\begin{array}{l}\text { Boys } \\
(n=104)\end{array}$ & $\begin{array}{l}p \text { value } \\
\text { क versus ơ }\end{array}$ & $\begin{array}{l}\text { Subgroup } \\
(n=41)\end{array}$ & $p$ value $^{\#}$ \\
\hline$(n=197)$ &
\end{tabular}

\section{Birth}

Gestational age (weeks)

Birth weight SDS

Birth length SDS

Age 1 month

Age (months)

Weight SDS

Length SDS

Sum of peripheral skinfolds (mm)

Sum of central skinfolds (mm)

Fat mass (kg)

Fat mass percentage (\%)

Age 3 months

Age (months)

Weight SDS

Length SDS

Sum of peripheral skinfolds (mm)

Sum of central skinfolds (mm)

Fat mass (kg)

Fat mass percentage $(\%)$

Visceral fat $(\mathrm{cm})$

Abdominal subcutaneous fat $(\mathrm{cm})$

Age 6 months

Age (months)

Weight SDS

Length SDS

Sum of peripheral skinfolds (mm)

Sum of central skinfolds (mm)

Fat mass (kg)

Fat mass percentage (\%)

Visceral fat $(\mathrm{cm})$

Abdominal subcutaneous fat $(\mathrm{cm})$

$\begin{array}{clcc}39.9 & 38.9 \text { to } 40.6 & 39.9 & 39.7 \\ -0.38 & -1.12 \text { to } 0.33 & -0.42 & -0.35 \\ 0.13 & -1.05 \text { to } 0.80 & 0.13 & -0.38 \\ & & & \\ 0.95 & 0.92 \text { to } 1.05 & 0.95 & 0.99 \\ 0.33 & -0.51 \text { to } 1.09 & 0.42 & 0.33 \\ 0.04 & -0.61 \text { to } 0.68 & 0.04 & 0.02 \\ 11.0 & 10.0 \text { to } 13.0 & 11.0 & 11.0 \\ 11.0 & 9.0 \text { to } 12.0 & 11.0 & 11.0 \\ 0.68 & 0.54 \text { to } 0.86 & 0.65 & 0.73 \\ 16.4 & 13.7 \text { to } 19.4 & 16.5 & 16.3 \\ & & & \\ 2.99 & 2.92 \text { to } 3.06 & 2.99 & 2.99 \\ 0.49 & -0.24 \text { to } 1.22 & 0.36 & 0.57 \\ 0.43 & -0.17 \text { to } 0.90 & 0.27 & 0.56 \\ 15.0 & 13.0 \text { to } 16.0 & 14.0 & 15.0 \\ 13.0 & 11.0 \text { to } 15.0 & 12.0 & 13.0 \\ 1.33 & 1.12 \text { to } 1.59 & 1.29 & 1.37 \\ 22.6 & 19.7 \text { to } 25.8 & 23.1 & 22.4 \\ 2.53 & 2.03 \text { to } 2.89 & 2.39 & 2.54 \\ 0.42 & 0.35 \text { to } 0.50 & 0.42 & 0.43 \\ & & & \\ 6.01 & 5.95 \text { to } 6.11 & 6.01 & 6.01 \\ 0.14 & -0.31 \text { to } 0.71 & 0.06 & 0.18 \\ 0.29 & -0.34 \text { to } 0.78 & 0.25 & 0.31 \\ 16.0 & 14.0 \text { to } 17.0 & 17.0 & 15.0 \\ 12.0 & 11.0 \text { to } 15.0 & 13.0 & 12.0 \\ 1.76 & 1.44 \text { to } 2.09 & 1.79 & 1.75 \\ 23.5 & 20.2 \text { to } 27.3 & 24.8 & 22.8 \\ 2.34 & 2.00 \text { to } 2.84 & 2.34 & 2.45 \\ 0.41 & 0.35 \text { to } 0.51 & 0.45 & 0.41\end{array}$

0.58

39.4

$\mathbf{0 . 0 3}$

0.60

$-0.85$

0.08

$0.47 \quad-0.97$

0.10

0.38

0.95

0.34

0.79

$-0.04$

0.09

0.61

$-0.11$

$\mathbf{0 . 0 3}$

0.73

0.33

0.67

0.46

0.62

0.65

0.39

0.49

$\begin{array}{cc}0.74 & 0.65 \\ 17.7 & 0.49\end{array}$

Data expressed as median (interquartile range)

Significant $P$ values are indicated in boldface. \# Differences between total group and subgroup

and 6 months (data not shown) (Table 4). A higher PYY at 3 months correlated with lower weight for length SDS at 3 months $(r=-0.23, p<0.01)$. Serum leptin and insulin at 3 months correlated with the increase in weight for length SDS between 1 and 3 months of life $(r=0.45, p<0.001$, $r=0.20, p<0.01$, resp.).

\section{Discussion}

In this study, we investigated the fasting serum levels of appetite-regulating hormones, such as ghrelin, leptin, insulin, GIP, PP and PYY in infants at 3 and 6 months and their associations with type of feeding, FM\%, visceral fat and subcutaneous fat. Interestingly, all appetite-regulating hormones were different between infants with formula feeding compared to the breastfed infants. Serum levels of ghrelin, leptin, insulin, GIP and PP were significantly higher in the formula-fed group, whereas PYY was significantly lower. Leptin at 3 months correlated positively with FM\% at 3 and 6 months, but only with subcutaneous fat and not with visceral fat. Also ghrelin at 3 months correlated with FM\% at 3 months, but only in formula-fed infants. Serum insulin correlated positively with gain in 
Table 2 Serum levels of appetite-regulating hormones in infants at 3 and 6 months of age

\begin{tabular}{|c|c|c|c|c|c|c|c|}
\hline & \multicolumn{4}{|c|}{3 months } & \multicolumn{2}{|c|}{6 months } & \multirow{2}{*}{$\begin{array}{l}p \text { value girls vs. } \\
\text { boys at } 3 \text { months }\end{array}$} \\
\hline & \multicolumn{2}{|l|}{ Girls } & \multicolumn{2}{|l|}{ Boys } & \multicolumn{2}{|l|}{ Boys } & \\
\hline \multicolumn{8}{|c|}{ Total group $(n=197)$} \\
\hline Hours fasting (h) & $2: 50$ & $2: 22-3: 30$ & $3: 12$ & $2: 30-3: 55$ & & & 0.11 \\
\hline Ghrelin (pg/ml) & 44.0 & $23.4-82.1$ & 48.9 & $23.4-68.3$ & & & 0.57 \\
\hline Leptin (pg/ml) & 1439.6 & $875.3-2364.7$ & 1405.2 & 742.6-2001.4 & & & 0.24 \\
\hline Insulin (pg/ml) & 369.0 & $232.3-619.5$ & 430.5 & $265.7-738.1$ & & & 0.30 \\
\hline $\mathrm{GIP}(\mathrm{pg} / \mathrm{ml})$ & 244.8 & $133.0-393.1$ & 237.3 & $138.5-350.8$ & & & 0.87 \\
\hline $\mathrm{PP}(\mathrm{pg} / \mathrm{ml})$ & 59.8 & $31.7-94.2$ & 68.3 & $46.5-104.1$ & & & 0.08 \\
\hline \multirow[t]{2}{*}{ PYY (pg/ml) } & 210.6 & $159.4-293.8$ & 196.9 & $156.3-250.8$ & & & 0.24 \\
\hline & & & & & & & $\begin{array}{l}p \text { value } 3 \text { versus } \\
6 \text { months }\end{array}$ \\
\hline \multicolumn{8}{|l|}{ Subgroup $(n=41)$} \\
\hline Hours fasting (h) & & & $3: 25$ & $2: 38-4: 00$ & 2.52 & $2.13-4.15$ & 0.252 \\
\hline Ghrelin (pg/ml) & & & 46.2 & $20.2-67.6$ & 83.1 & $48.7-102.6$ & $<0.001$ \\
\hline Leptin (pg/ml) & & & 1560.4 & 945.4-1991.4 & 827.2 & $424.6-1247.2$ & $<0.001$ \\
\hline Insulin (pg/ml) & & & 464.9 & $291.6-718.5$ & 483.8 & $324.4-635.6$ & 0.821 \\
\hline GIP (pg/ml) & & & 263.1 & $137.1-345.4$ & 310.9 & $201.5-421.1$ & 0.008 \\
\hline $\mathrm{PP}(\mathrm{pg} / \mathrm{ml})$ & & & 70.9 & $41.4-127.8$ & 115.7 & $68.4-241.6$ & $<0.001$ \\
\hline PYY (pg/ml) & & & 171.6 & $150.1-231.7$ & 158.7 & $130.7-205.6$ & 0.065 \\
\hline
\end{tabular}

Data expressed as median (interquartile range)

Significant $P$ values are indicated in boldface

Table 3 Serum levels of appetite-regulating hormones in 197 infants at 3 months, divided by type of feeding

\begin{tabular}{lccr}
\hline & $\begin{array}{l}\text { Exclusive FF at } \\
\text { 3 months }\end{array}$ & $\begin{array}{l}\text { Exclusive BF at } \\
3 \text { months }\end{array}$ & $p$ value \\
\hline Ghrelin $(\mathrm{pg} / \mathrm{ml})$ & 49.1 & 33.2 & $\mathbf{0 . 0 2 6}$ \\
Leptin $(\mathrm{pg} / \mathrm{ml})$ & 1719.1 & 1190.1 & $\mathbf{0 . 0 1 8}$ \\
Insulin $(\mathrm{pg} / \mathrm{ml})$ & 560.9 & 287.8 & $<\mathbf{0 . 0 0 1}$ \\
GIP $(\mathrm{pg} / \mathrm{ml})$ & 304.1 & 198.5 & $\mathbf{0 . 0 0 2}$ \\
PP $(\mathrm{pg} / \mathrm{ml})$ & 87.7 & 46.3 & $<\mathbf{0 . 0 0 1}$ \\
PYY $(\mathrm{pg} / \mathrm{ml})$ & 180.2 & 231.0 & $\mathbf{0 . 0 0 2}$ \\
\hline
\end{tabular}

Data were expressed as medians. Significant $P$ values are indicated in boldface
FM\% between 1 and 3 months. Other appetite-regulating hormones were not associated with fat mass percentage and visceral fat in the first 3-6 months.

We show that formula-fed infants have different levels of appetite-regulating hormones than infants with exclusive breastfeeding. For healthy term infants, exclusive breastfeeding is considered the reference to which formula feeding must be compared. Serum levels of ghrelin were significantly higher in formula fed than in breastfed infants. These data are in line with a cross-sectional study where the same results were found in the first year of life in a group of Italian infants [20]. It has been demonstrated in animal studies that neonatal ghrelin is important for normal maturation

Table 4 Correlations of appetite-regulating hormones with body composition and anthropometry at 3 months in 197 infants

\begin{tabular}{lcccccc}
\hline & FM\% & $\begin{array}{l}\text { Delta FM\% 1-3 } \\
\text { months }\end{array}$ & Visceral fat & Subcutaneous fat & $\begin{array}{l}\text { Weight for length } \\
\text { SDS }\end{array}$ & $\begin{array}{l}\text { Delta weight for length } \\
\text { SDS 1-3 months }\end{array}$ \\
\hline Ghrelin (pg/ml) & 0.11 & 0.01 & $<0.01$ & $<0.01$ & 0.06 & 0.04 \\
Leptin (pg/ml) & $\mathbf{0 . 3 7 * *}$ & 0.04 & 0.01 & $0.16^{\wedge}$ & $\mathbf{0 . 4 8}^{* *}$ & $\mathbf{0 . 4 5}^{* *}$ \\
Insulin (pg/ml) & $<0.01$ & $\mathbf{0 . 2 2} *$ & $0.19^{*}$ & -0.01 & 0.08 & $\mathbf{0 . 2 0}$ \\
GIP (pg/ml) & 0.03 & $-0.16^{\wedge}$ & 0.11 & -0.08 & 0.04 & 0.13 \\
PP (pg/ml) & 0.04 & -0.02 & 0.04 & -0.02 & 0.03 & 0.09 \\
PYY (pg/ml) & -0.08 & 0.03 & 0.03 & -0.12 & $-\mathbf{0 . 2 3}^{*}$ & -0.12 \\
\hline
\end{tabular}

Significant $P$ values are indicated in boldface if $r>0.20$ and $p<0.05 ;{ }^{\wedge} p<0.05 ; * p<0.01 ; * * p<0.001$ 
of hypothalamic neural circuits. The neural developmental activity of ghrelin is essentially restricted to the neonatal period [21]. Proper expression of ghrelin during neonatal life is, therefore, crucial for lifelong metabolic regulation, and too high ghrelin levels during the first months of life result in lifelong metabolic disturbances [22]. In the formula-fed infants, we did not find very high levels of ghrelin, but their levels were significantly higher than in the breastfed infants. In this critical period, this could have adverse effects on the maturation of the hypothalamic neural circuits, resulting in less favorable metabolic regulation in later life [23]. We found also higher leptin levels in formula-fed infants. Like ghrelin, leptin is known to promote the development of the arcuate nucleus in the hypothalamus, and its developmental action is also restricted to a critical window in the first months of life [24], but higher ghrelin levels could impair leptin signaling in the arcuate nucleus in early life [21]. Our data are in line with another study, reporting higher leptin levels in formula-fed infants compared to breastfed infants [25]. However, in a study in infants with a smaller group of subjects, breastfed infants had higher leptin levels than formula-fed infants [26].

Infants with formula feeding had lower levels of PYY. One of the actions of PYY is to reduce food intake by reducing the gastro-intestinal tract motility and the gastric emptying, via the vagal-brainstem-hypothalamic pathway [27]. As the breastfed infants had higher PYY levels, that could be a link in the protective role of breastfeeding for obesity. In addition, we found that higher levels of PYY were associated with a lower weight for length SDS at 3 months. These results are in line with the data of Helsinki Birth Cohort Study, where they found higher PYY levels in adulthood when the infants had a lower growth rate during infancy [28]. Our study shows that these differences can already be found in early life. Infants with formula feeding had also higher levels of insulin, GIP and PP. A possible explanation of the higher insulin levels in formula-fed infants could be the amount of proteins, which is in general higher in formula feeding than in breastfeeding [29]. Another higher appetite-regulating hormone in formula-fed infants was GIP, which plays an important role in the regulation of plasma glucose and insulin secretion [30]. It is possible that higher GIP levels could account for an enhanced insulin release [31]. Also PP was higher in formula-fed infants. The function of PP is to self-regulate pancreatic secretion activities [32]. However, the mechanism of how the type of feeding influences the GIP and PP levels is not fully understood. For further interpretation of the potential relevance of these findings, more research is required.

To our knowledge, this is the first large study investigating the relationship between simultaneously measured serum levels of appetite-regulating hormones and detailed measurements of infant body composition, by air displacement plethysmography (Peapod). We found a significant positive correlation between serum ghrelin and FM\% at 3 months in the formula-fed infants, but not in the breastfed group. Leptin was also correlated with FM\% in both formula-fed and breastfed groups. The associations of leptin with FM\% could be explained by the known physiology of adipose tissue. During early life, the adipocytes proliferate and differentiate [33]. Leptin levels increase in parallel to the number of adipocytes during the last part of the third trimester during gestation and the first months of life [34]. We demonstrated that leptin levels in male infants were higher at 3 months than at 6 months. Serum insulin correlated positively with gain in FM\% between 1 and 3 months. Earlier studies showed a positive association of insulin and insulin resistance with fat mass in childhood and adulthood $[35,36]$. The other appetite-regulating hormones such as PP and GIP did not associate with FM\% or gain in FM\% in the total group, which partly contrasted our expectations. There is a need for further studies to identify the underlying mechanisms and potential implications for possible programming effects of serum appetite hormones on later body composition.

We could not identify differences in appetite-regulating hormones between boys and girls, which is in line with studies in older children and in a study with a subset of appetite-regulating hormones in infants [30, 37, 38].

We did not investigate hormone levels in breast milk. Some studies investigated levels of ghrelin, leptin or insulin in breast milk [9, 39-42]. In these studies, associations between appetite-regulating hormones in breast milk and anthropometric data of infants were found. An unhealthy maternal diet can lead to an unfavorable appetite-regulating hormone profile in breast milk, but it is not clear by which mechanism this unfavorable profile in breast milk could influence infant growth as hormones will be degraded when passing the stomach. In our study, we only investigated fasting levels of appetite-regulating hormones. We realize that several appetite-regulating hormones are low in fasting state [30], but our study shows that even in fasting state all hormones were different between the formula-fed and breastfed infants. It would be interesting to investigate appetite-regulating hormone levels before and after a meal, but in our study, it was found unethical to collect blood for research purpose twice in healthy infants.

In conclusion, we found significantly higher serum levels of ghrelin, leptin, insulin, GIP and PP and lower serum levels of PYY in infants who received exclusive formula feeding for at least 3 months compared to breastfeeding. Besides associations of leptin, ghrelin and insulin with body composition in infants of 3 and 6 months of age, we could not identify other associations of serum appetite-regulating hormones with body composition. Further 
studies on appetite-regulating hormones and the interactions between these and body composition later in infancy would be helpful to further elucidate whether appetite-regulating hormones contribute to the programming of metabolic health in later life.

Acknowledgments We thank all the patients and their parents for participating in this study. We greatly acknowledge Mrs. J. van Nieuwkasteele, M. Huibregtse-Schouten and C. Bruinings-Vroombout, research nurses, for their assistance and help with the data collection. We also thank L. van der Zee for analyzing the appetite-regulating hormones. Besides this, we thank Nutricia Research for their independent research grant. This study was an investigator-initiated study, and AHK received an independent research grant by Nutricia Research, The Netherlands.

\section{Compliance with ethical standards}

Conflict of interest The authors have no disclosures or conflict of interest to declare.

Open Access This article is distributed under the terms of the Creative Commons Attribution 4.0 International License (http://creativecommons.org/licenses/by/4.0/), which permits unrestricted use, distribution, and reproduction in any medium, provided you give appropriate credit to the original author(s) and the source, provide a link to the Creative Commons license, and indicate if changes were made.

\section{References}

1. Statistiek, C.B.v.d. Lengte en gewicht van personen, ondergewicht en overgewicht; vanaf 1981 (Website) 201225 October 2012 [cited 201203 July 2012]. http://statline.cbs.n1/StatWeb/publicati on $/$ DM $=$ SLNL $\& \mathrm{PA}=81565 \mathrm{NED} \& \mathrm{D} 1=\mathrm{a} \& \mathrm{D} 2=0,1 \& \mathrm{D} 3=\mathrm{a} \& \mathrm{D} 4$ $=0 \& \mathrm{D} 5=0,10,20,30 \& \mathrm{HDR}=\mathrm{T} \& \mathrm{STB}=\mathrm{G} 1, \mathrm{G} 2, \mathrm{G} 3, \mathrm{G} 4 \& \mathrm{VW}=\mathrm{T}$

2. Singh AS et al (2008) Tracking of childhood overweight into adulthood: a systematic review of the literature. Obes Rev 9(5):474-488

3. Tsiros MD et al (2011) Obesity: the new childhood disability? Obes Rev 12(1):26-36

4. Reilly JJ (2006) Obesity in childhood and adolescence: evidence based clinical and public health perspectives. Postgrad Med J 82(969):429-437

5. Leunissen RW et al (2009) Timing and tempo of first-year rapid growth in relation to cardiovascular and metabolic risk profile in early adulthood. JAMA 301(21):2234-2242

6. Ekelund $U$ et al (2007) Association of weight gain in infancy and early childhood with metabolic risk in young adults. J Clin Endocrinol Metab 92(1):98-103

7. Eriksson JG (2011) Early growth and coronary heart disease and type 2 diabetes: findings from the Helsinki Birth Cohort Study (HBCS). Am J Clin Nutr 94(6 Suppl):1799S-1802S

8. Munzberg H, Morrison CD (2015) Structure, production and signaling of leptin. Metabolism 64(1):13-23

9. Savino F et al (2008) Looking for a relation between serum leptin concentration and body composition parameters in healthy term infants in the first 6 months of life. J Pediatr Gastroenterol Nutr 46(3):348-351

10. Maric $\mathrm{G}$ et al (2014) The role of gut hormones in appetite regulation (review). Acta Physiol Hung 101(4):395-407

11. Heijboer AC et al (2006) Gut-brain axis: regulation of glucose metabolism. J Neuroendocrinol 18(12):883-894
12. Baggio LL, Drucker DJ (2007) Biology of incretins: GLP-1 and GIP. Gastroenterology 132(6):2131-2157

13. Iniguez $\mathrm{G}$ et al (2002) Fasting and post-glucose ghrelin levels in SGA infants: relationships with size and weight gain at one year of age. J Clin Endocrinol Metab 87(12):5830-5833

14. Schonbeck Y et al (2011) Increase in prevalence of overweight in Dutch children and adolescents: a comparison of nationwide growth studies in 1980, 1997 and 2009. PLoS One 6(11):e27608

15. Ellis KJ et al (2007) Body-composition assessment in infancy: air-displacement plethysmography compared with a reference 4-compartment model. Am J Clin Nutr 85(1):90-95

16. $\mathrm{Ma} \mathrm{G}$ et al (2004) Validation of a new pediatric air-displacement plethysmograph for assessing body composition in infants. Am J Clin Nutr 79(4):653-660

17. Sainz RD, Urlando A (2003) Evaluation of a new pediatric airdisplacement plethysmograph for body-composition assessment by means of chemical analysis of bovine tissue phantoms. Am J Clin Nutr 77(2):364-370

18. Urlando A, Dempster P, Aitkens S (2003) A new air displacement plethysmograph for the measurement of body composition in infants. Pediatr Res 53(3):486-492

19. De Lucia Rolfe E et al (2013) Ultrasound estimates of visceral and subcutaneous-abdominal adipose tissues in infancy. J Obes 2013:951954

20. Savino F et al (2005) Ghrelin, leptin and IGF-I levels in breastfed and formula-fed infants in the first years of life. Acta Paediatr 94(5):531-537

21. Steculorum SM et al (2015) Neonatal ghrelin programs development of hypothalamic feeding circuits. J Clin Invest 125(2):846-858

22. Collden $\mathrm{G}$ et al (2015) Neonatal overnutrition causes early alterations in the central response to peripheral ghrelin. Mol Metab $4(1): 15-24$

23. Hayashida $\mathrm{T}$ et al (2002) Ghrelin in neonatal rats: distribution in stomach and its possible role. J Endocrinol 173(2):239-245

24. Bouret SG, Draper SJ, Simerly RB (2004) Trophic action of leptin on hypothalamic neurons that regulate feeding. Science 304(5667): 108-110

25. Petridou E et al (2005) Neonatal leptin levels are strongly associated with female gender, birth length, IGF-I levels and formula feeding. Clin Endocrinol (Oxf) 62(3):366-371

26. Savino F et al (2004) Breast-fed infants have higher leptin values than formula-fed infants in the first four months of life. J Pediatr Endocrinol Metab 17(11):1527-1532

27. Wojcicki JM (2012) Peptide YY in children: a review. J Pediatr Endocrinol Metab 25(3-4):227-232

28. Perala MM et al (2013) Early growth and postprandial appetite regulatory hormone responses. Br J Nutr 110(9):1591-1600

29. Ziegler EE (2006) Growth of breast-fed and formula-fed infants. Nestle Nutr Workshop Ser Pediatr Prog 58:51-59

30. Huml M et al (2011) Gut peptide hormones and pediatric type 1 diabetes mellitus. Physiol Res 60(4):647-658

31. Le Huerou-Luron I, Blat S, Boudry G (2010) Breast-v. formulafeeding: impacts on the digestive tract and immediate and longterm health effects. Nutr Res Rev 23(1):23-36

32. Batterham RL et al (2003) Pancreatic polypeptide reduces appetite and food intake in humans. J Clin Endocrinol Metab 88(8):3989-3992

33. Hernandez MI et al (2012) Leptin and IGF-I/II during the first weeks of life determine body composition at 2 years in infants born with very low birth weight. J Pediatr Endocrinol Metab 25(9-10):951-955

34. Granado M et al (2012) Leptin in early life: a key factor for the development of the adult metabolic profile. Obes Facts 5(1):138-150 
35. Gishti $\mathrm{O}$ et al (2015) BMI, total and abdominal fat distribution, and cardiovascular risk factors in school-age children. Pediatr Res 77(5):710-718

36. Paradisi G et al (1999) Dual energy X-ray absorptiometry assessment of fat mass distribution and its association with the insulin resistance syndrome. Diabetes Care 22(8):1310-1317

37. Fidanci $\mathrm{K}$ et al (2010) Ghrelin levels and postnatal growth in healthy infants $0-3$ months of age. J Clin Res Pediatr Endocrinol 2(1):34-38

38. Wilasco MI et al (2012) Ghrelin, leptin and insulin in healthy children: Relationship with anthropometry, gender, and age distribution. Regul Pept 173(1-3):21-26
39. Fields DA, Demerath EW (2012) Relationship of insulin, glucose, leptin, IL-6 and TNF-alpha in human breast milk with infant growth and body composition. Pediatr Obes 7(4):304-312

40. Kon IY et al (2014) The study of breast milk IGF-1, leptin, ghrelin and adiponectin levels as possible reasons of high weight gain in breast-fed infants. Ann Nutr Metab 65(4):317-323

41. Savino F et al (2012) Resistin and leptin in breast milk and infants in early life. Early Hum Dev 88(10):779-782

42. Savino F et al (2010) Evaluation of leptin in breast milk, lactating mothers and their infants. Eur J Clin Nutr 64(9):972-977 